\title{
Glass bead filled Polyetherketone (PEK) composite by High Temperature Laser Sintering (HT-LS)
}

Yuan Wang ${ }^{a *}$, Edward James ${ }^{b}$, Oana R. Ghita ${ }^{a}$

a.University of Exeter, Department of Engineering, Mathematics and Physical Sciences, North Park Road, EX4 4QF, UK

b. Centek Limited, Station View, Forde Road, Brunel Industrial Estate, Newton Abbot, Devon UK, TQ12 4AE

*Corresponding author: y.wang@exeter.ac.uk Tel: +44-1392-725779

\begin{abstract}
Thermal expansion behavior and mechanical performance of high temperature laser sintering PEK (LS-PEK) and glass bead filled PEK composite (LS-GB/PEK) are reported and discussed for the first time. The laser sintered PEK and its composite show anisotropic thermal expansion properties related with their multilayer structure created by the laser sintering method. Compared with the PEK manufactured by conventional injection molding (IM-PEK), LS-PEK has a 10\% lower tensile strength but a higher hardness due to its greater crystallinity. Addition of glass bead to PEK increases the hardness of PEK without affecting the ultimate tensile strength and also improves the thermal stability of laser sintered parts. The rule of mixtures is applied to simulate the coefficient of thermal expansion (CTE) of the LS-GB/PEK composite structures and compare with experimental results. The measured CTE values match the calculated results below $\mathrm{Tg}$ and deviate slightly from the simulated trend line above $\mathrm{Tg}$. Keywords: Laser Sintering, Glass, Polyetherketone, Thermal analysis, Thermal expansion, Microstructure
\end{abstract}




\section{Introduction}

The family of Polyaryletherketone (PAEK) polymers includes high temperature, high performance semi-crystalline polymers with excellent mechanical performance, high wear and chemical resistance such as PEEK, PEK and PEKK. ${ }^{[1,2,3]}$ Due to their unique characteristics, these polymers and their composites have been extensively investigated in terms of structure, properties and processing ${ }^{[4,5,6,7,8]}$ and have been used in automotive, aerospace and medical applications using injection and compression moulding as the main manufacturing methods. ${ }^{[9,10,11]}$ For example, the aerospace sector is becoming increasingly interested in thermoplastic composites: several parts have been used over the last few years on A340 and A380 structures. ${ }^{[12,13,14]}$ Similarly, in the automotive sector, the increased pressure for reusable, sustainable and lightweight structures makes these polymers and their composites increasingly attractive as alternative materials to the standard thermosetting materials ${ }^{[12,13]}$ for body parts and as metal replacement components for the future engine blocks. ${ }^{[15,16]}$ One of the heaviest components of a car is the engine block, typically made of steel and aluminum. The need to reduce weight in all types of transport vehicles will inevitably lead to plastic engines. ${ }^{[15]}$ Such plastic engines will have to withstand harsh conditions such as high operating temperatures and highly corrosive media, therefore high temperature polymers and composites will be at the heart of such developments and properties such as thermal conductivity, thermal expansion and wear will become increasingly important. In an attempt to push further the limit of materials, design and applications, this paper presents for the first time a characterization study of glass bead filled PEK composites manufactured using high temperature laser sintering (HT-LS) with particular attention to their thermal expansion behavior. As with the majority of the 
additive manufacturing processes, one of the advantages offered by the LS process is the freedom of design which can fabricate highly complex structures unachievable any other way. The combination of high performance materials with LS as manufacturing process is highly radical and can open a wide range of opportunities in high temperature composite applications. Due to their high service temperature and high thermal stability, glass-PEEK materials have received significant attention in the literature. ${ }^{[17,18,19]}$ Incorporation of glass into PEEK has been mostly studied for bearing applications as sliding materials where glass is added primarily as non-reinforcing filler ${ }^{[20,21]}$ to achieve low friction and wear rates. Metal replacement materials with low CTE had been also previously created ${ }^{[22]}$ using thermosetting resins with $75 \%$ glass, and additionally reinforced with $0.5 \%$ Twaron short fibers. The composite created at such high glass loadings showed interesting properties with a CTE of approximately $15 \mathrm{ppm} \mathrm{k}^{-1}$ and a $\operatorname{Tg}$ of $340^{\circ} \mathrm{C}$.

Review of literature showed that the majority of studies in the PAEK polymer family had been carried out on PEEK rather than PEK or PEKK polymers. Although both polymers offer overall improved performance, the difference in chemical structure leads to higher melting temperatures and glass transition temperatures for $\mathrm{PEK}\left(\mathrm{Tm}=372{ }^{\circ} \mathrm{C}\right.$, $\left.\mathrm{Tg}=164{ }^{\circ} \mathrm{C}\right)$ over PEEK $\left(\mathrm{Tm}=343^{\circ} \mathrm{C}, \mathrm{Tg}=143^{\circ} \mathrm{C}\right) .{ }^{[3]}$ The lower melting and glass transition temperatures of PEEK allowed its easier implementation in manufacturing processes and therefore it was preferred over PEK, even though, ultimately, PEK is a stronger material. Although the primary aim of this study was to further improve thermal stability of PEK through incorporation of glass beads, the new material will also have cost benefits (reduced price of the resulting LS component due to less use of PEK material). 


\section{Manufacturing}

\subsection{Preparation of powder for high temperature laser sintering}

The HP3 PEK powder supplied by EOS (melt viscosity =190Pa.s ) was mixed with Potters Spheriglass 2000 CP3 glass beads in a 80/20\% (PEK/Glass) by weight mixing ratio. The glass beads used in this study is solid glass beads. The mixing of glass and PEK powder was carried out using an EOS electric powder roller mixer for 1 hour in a 15 litre drum.

\subsection{High temperature laser sintering}

The LS process was carried out in a reduced build mode using the EOSINT P800 system. ${ }^{[23]}$ The parts were built in the shape of dog bone geometries $80 \times 10 \times 4 \mathrm{~mm}$, according to ISO527-2 test specimen type 1BA. The position of the parts within the build chamber is shown in Figure 1. The system went through a warm up stage where it gradually increased the temperature of the base powder to the $368^{\circ} \mathrm{C}$ process temperature under an inert gas atmosphere. After completion of the warm up stage the EOSINT P800 started the laser sintering build stage. During the laser sintering process the powder was applied to the bed by means of a recoating mechanism to give a layer thickness of $0.12 \mathrm{~mm}$. The parts were then scanned using the $\mathrm{CO}_{2}$ laser at $15 \mathrm{~W}$ power and $2250 \mathrm{~mm} \mathrm{~s}^{-1}$ scanning speed. Once sintered, the material went through a post-cure stage where the powder layer was exposed to thermal radiation for a further 12 seconds to enhance the fusion between powder grains. The piston then dropped by the layer thickness and the recoating mechanism applied the next layer of powder. On completion of the build the machine entered a cool down stage. The parts were then cooled down to room temperature. ${ }^{[23]}$ 


\subsection{Injection molding}

The material used for injection moulding was VICTREX® ${ }^{\circledR}$ HT G22 pellets (melt viscosity $=220 \mathrm{~Pa} . \mathrm{s}$ ), According to manufacture, VICTREX ${ }^{\circledR} \mathrm{HT}$ G22 is equivalent to EOS PEK HP3. The pellets were dried for 5 hours at $120^{\circ} \mathrm{C}$. Dog bone samples were manufactured as per manufacturer specifications (see section 2.2). The nozzle temperature was $400^{\circ} \mathrm{C}$, injection hydraulic pressure was 900 bars for 2 seconds, the holding hydraulic pressure was 900 bars; injection speed was $60 \mathrm{~cm}^{3} \mathrm{~s}^{-1}$ and the cooling time was 15 seconds. The injection moulding samples were used for benchmarking.

\section{Experimental Methods}

\subsection{Scanning Electron Microscopy (SEM)}

Microstructure analysis of glass beads, PEK powders and the fracture surface of samples were carried out by secondary electron microscopy SEM (Hitachi S-3200N, Japan). The secondary electron images were taken under $25 \mathrm{Kv}$ acceleration voltage in the low vacuum chamber with pressure of $50 \mathrm{~Pa}$.

\subsection{Wide Angle X-Ray Diffractometry (WAXD)}

The WAXD measurements were performed with a Bruker D8 Advance WAXD with copper anode at room temperature. XRD data were collected in the angular range where $2 \theta=10^{\circ}-32^{\circ}$. The step size of $2 \theta$ was $0.01^{\circ} . \mathrm{Al}_{2} \mathrm{O}_{3}$ was used as a standard sample to measure the instrumental line broadening and to calibrate the diffractometer. The percentage relative crystallinity $(\% X c)$ of various samples and their lamellar thickness $(L)$ were calculated from equations (1) and (2):

$$
X_{c}=\left[A_{c} /\left(A_{c}+A_{a}\right)\right] \times 100 \%
$$


$L=K \lambda / F W_{(s)} \cos \theta$

where $A_{c}$ is area of crystalline phase, $A_{s}$ is area of amorphous phase, and $\lambda$ is the wavelength of the copper anode $(0.154 \mathrm{~nm}), K$ is constant $(K=1)$ and $F W_{(s)}$ is the specimen peak broadening at half the maximum intensity (FWHM) in radians.

\subsection{Thermal Expansion Measurements}

For thermal expansion tests, the dog bone shaped parts were cut down into cubic specimens of approximate $3 \times 3 \times 3 \mathrm{~mm}$. The measurements were made in all three directions $\mathrm{X}, \mathrm{Y}$ and $\mathrm{Z}$ in order to investigate the thermal expansion dependence of the building direction. For LS samples, the $\mathrm{X}, \mathrm{Y}$ and $\mathrm{Z}$ directions as a function of the building direction are shown in Figure 1. For the injection moulded samples, $X, Y$ and $\mathrm{Z}$ directions represent the width, length and thickness of the sample, respectively. For each type of sample, three repeat tests were performed on three different specimens. Specimens were annealed in oven for $1 \mathrm{hr}$ at $160^{\circ} \mathrm{C}$ to release any internal stress before conducting thermal expansion measurements. Thermal expansion tests were conducted in a Mettler Toledo TMA 40. A negligible force of $0.002 \mathrm{~N}$ was applied using a $3 \mathrm{~mm}$ flat quartz probe. To obtain the accurate coefficient of thermal expansion, two heating cycles were applied on each specimen. In the first heating cycle, specimens were heated from $30{ }^{\circ} \mathrm{C}$ to $300^{\circ} \mathrm{C}$ at a heating rate of $5{ }^{\circ} \mathrm{C} \mathrm{min}^{-1}$ in air, held at $300{ }^{\circ} \mathrm{C}$ for 10 minutes, then cooled down to $50{ }^{\circ} \mathrm{C}$ at $10 \circ \mathrm{C} \mathrm{min}^{-1}$ cooling rate. During the second heating cycle, the specimens were heated from $50{ }^{\circ} \mathrm{C}$ to $300{ }^{\circ} \mathrm{C}$ at $5{ }^{\circ} \mathrm{C} \mathrm{min}^{-1}$ in air. The thermal scan recorded during the second heating cycles were used to calculate the CTE below glass transition temperature, $\alpha_{\mathrm{g}}$, and the CTE above glass transition temperature, $\alpha_{\gamma}$. 


\subsection{Mechanical testing}

The tensile tests were performed on a Lloyds EZ20 machine with a crosshead speed of $2 \mathrm{~mm} \mathrm{~min}^{-1}$ for all samples. 20 samples were tested.

Hardness testing was performed on a digital micro-hardness tester FM-1e (FutureTech Crop. Japan) the indentation force used was 300gf, 10s. The lengths of diagonal left by the indenter were measured, and the Vickers hardness (HV) was calculated from equation (3):

$H V=1.854 F / d^{2}$

Where $F$ is the force in Kgf applied to the diamond and $d$ is the average length of the diagonal left by the indenter in $\mathrm{mm}$.

\section{Results and discussion}

\subsection{Characterization of the raw materials}

Figure 2 (a) and (b) shows the SEM image of glass beads and PEK powder, respectively. The glass beads have almost perfect spherical shape, while the PEK powders have a flake like appearance. Previous particle analysis ${ }^{[24]}$ of HP3 PEK powder showed that $50 \%$ volume frequency had particle size in range of $37.5-63 \mu \mathrm{m}$ whereas the glass beads, $52 \%$ volume frequency had diameter in range of $16-27.5 \mu \mathrm{m}$. In spite of its irregular shape, the HP3 PEK powder dispersed well during manufacture. The addition of the glass beads however improved further the flow and spreading characteristics of powder in laser sintering process.

\subsection{Mechanical performance}

Figure 3(a) and (b) show the stress-strain curve, tensile strength and hardness of injection moulded PEK specimens and laser sintered PEK and GB/PEK composite. For 
comparison purposes, the ultimate tensile strength (UTS) and Vickers hardness results of IM- PEK, LS-PEK and LS-GB/PEK composite are listed in Table 1(a). Amongst the three types of samples, the IM-PEK had the highest UTS but the lowest Vickers hardness. All laser sintered specimen show less percentage of strain than injection moulded ones at break. Addition of the glass beads to the PEK powder in the laser sintering process neither reduced nor increased its tensile strength. This suggests a good interfacial bonding between the glass beads and the matrix and indicates that the glass beads used were not at a critical size distribution or weight fraction to influence positively or negatively the failure mechanisms and the strength. ${ }^{[25]}$ The variance of UTS was great for laser sintered samples, as they were built along various directions.

The LS-GB/PEK composite showed a 7\% increase in hardness when compared with LS-PEK and a $24 \%$ increase when compared with IM-PEK. The presence of the glass beads increased the hardness of the LS-GB/PEK composite; the hardness values obtained from the composite representing an average measurement of the manufactured samples incorporating both components (glass and PEK). It is interesting to notice that depending on the manufacturing process applied, the hardness of the same material (PEK) is significantly different, LS-PEK having an approximately $18 \%$ increase in hardness compared with IM-PEK samples. This is believed to be the result of the two very different cooling regimes introduced by the two manufacturing processes which lead to different crystallinities and crystal morphology. For example, Stuart and Briscoe ${ }^{[26]}$ noticed that the hardness of crystalline PEEK is significantly higher than that of the amorphous PEEK material, while Lu and Friedrich ${ }^{[18]}$ also concluded that the spherulite size of PEEK influenced the hardness and ultimately the friction and wear performance. 


\subsection{Microstructure of LS-PEK and LS-GB/PEK composite}

\subsubsection{LS-PEK}

The fracture surface of LS-PEK in Figure 4(a)-(d) show a multi-layered structure consisting of two distinct morphologies: (1) a homogeneously sintered structure approximately $80 \mu \mathrm{m}$ thick, where the presence of individual particles is lost completely and (2) a dense and fully sintered layer where the presence of individual particles is still clearly visible with an average thickness of $40 \mu \mathrm{m}$. For clarity and ease of discussion, the two morphologies will be referred to as melted layer 1 and particle layer 2 . Considering that the combined thickness of the two layers is approximately $120 \mu \mathrm{m}$, it is assumed that layer 1 and layer 2 form one LS layer spread during the sintering process. Figure 4 (e) gives a schematic representation of this phenomenon. A similar structure (Figure 5) has been noticed by Zarringhalam et al., ${ }^{[27]}$ when investigating the microstructure of laser sintered nylon 12. The authors referred to 'unmelted' particle cores surrounded by melted and crystallised region" when investigating particles noticed here in particle layer 1 . The authors were able to correlate the layering effect with a double melting peak in the DSC thermograms, where a high peak represented large amounts of un-melted material, and a low peak was representative of a more complete melting. They also introduced the terminology of "degree of particle melt (DPM)" when discussing the layering effect similar to the one noticed here. Ajoku et al., ${ }^{[28]}$ explained clearly the bonding mechanisms and temperature differences that take place between vectors and layers and led to the layered structure. The neck growth is significantly smaller in $\mathrm{Z}$ direction than $\mathrm{X}$ and $\mathrm{Y}$ axes. Once a layer has been sintered and a new layer of powder is applied, the particles in the previous layer cooled to such an extent that bonding between particles in different layers is significantly lower than 
the particle bonding between or within vectors. Due to this reason, increasing energy input can help diminish creation of large differences between $\mathrm{X}, \mathrm{Y}$ and $\mathrm{Z}$ but cannot eliminate them completely. The layered structure and weaker mechanical performance in $\mathrm{Z}$ direction is an inherent effect of the manufacturing process. Nylon, a wellresearched and optimised polymeric material for laser sintering showed a difference of $16 \%$ in tensile strength in the $\mathrm{X}, \mathrm{Y}$ and $\mathrm{Z}$ axes. ${ }^{[28]}$ Previous study on laser sintered HP3 PEK showed a significantly higher difference in tensile strength amongst the three directions ${ }^{[29]}$ (approx. 50\%), which could the result of the higher processing temperatures and therefore higher thermal gradients between layers in $\mathrm{Z}$ axis.

\subsubsection{LS-GB/PEK composite}

The fractured surface of the LS-GB/PEK composites presented in Figure 1(c), shows a good interface bonding between the glass beads and PEK matrix, the glass beads were embedded in the polymer matrix without being damaged or pulled out. The coating on the glass beads is designed to promote the adhesion with polymers with polar function groups such as ether, esters, amides, ketones, and sulfone. ${ }^{[30]}$ PEK have ether and ketones groups therefore it is compatible with the coating on the glass beads.

\subsection{Crystallinity and Morphology}

Figure 6 presents the WAXD spectra of the injection moulded PEK and high temperature laser sintered PEK and GB/PEK composite samples. Reflections of crystal planes ${ }^{[31]} 110,111,200,112,211$ and 020 are indexed in the figure. Table 1(b) shows the peak position of crystal planes and half the maximum intensity (FWHM) for each type of sample. Increasing of FWHM in IM sample indicates a lower degree of crystalline perfection and smaller crystal dimension compared with the LS sample. 
The crystallinity and lamellar thickness values obtained from WAXD are shown in

Table 1(a). An approximately $40 \%$ increase in lamellar thickness and $24 \%$ increase in crystallinity from the injection moulding samples to laser sintered samples can be seen. The results are not surprising considering the difference in cooling rate applied in the two processes: injection moulding processes requires typically cooling times of few seconds, whereas the cooling in laser sintering process takes approximately $36 \mathrm{~h}$ at an average rate of $0.2{ }^{\circ} \mathrm{C} \mathrm{min}^{-1} .{ }^{[23]}$ Ghita et al., ${ }^{[23]}$ noticed an approximately $20 \%$ increase in crystallinity between the virgin and used PEK powders used in the high temperature laser sintering process. The used powder experienced a similar cooling process as the samples manufactured in this study. The presence of the glass beads had no significant influence on the crystallinity and the lamellar thickness of laser sintered PEK as shown in Table 1(a). Similarly, Sarasua et al., ${ }^{[32]}$ found that increasing the glass fibre content of PEEK composites (from 10 to $30 \%$ GF) does not significantly affect the crystallinity.

\subsection{Thermal expansion}

Table 1(a) presents the thermal expansion measurements of the injection moulded and high temperature laser sintered samples above and below the glass transition temperature as a function of the building direction. The increase in temperature through the glass transition led to a significant increase in CTEs for laser sintered and injection moulded samples due to increase of molecular movement. The anisotropic coefficient of thermal expansion was found in laser sintered samples, LS-PEK and LS-GB/PEK composite. Their thermal expansion in $\mathrm{X}$ and $\mathrm{Y}$ directions were similar but higher than those in $\mathrm{Z}$ direction. It could be that the layering effect restricts the free expansion across $\mathrm{Z}$ direction, the layers expanding against each other, creating some internal compression stress. In contrast, along X or Y direction, each layer has the same 
direction of thermal expansion, therefore more freedom to expand. Both thermal expansion values of the LS-GB/PEK composite below and above the glass transition $\left(\alpha_{\mathrm{g}}\right.$ and $\alpha_{\mathrm{r}}$ ) were lower than the LS-PEK. The presence of glass beads in the matrix helped lower the overall thermal expansion of LS-GB/PEK composite.

\subsection{Modelling and validation of CTE in LS-GB/PEK composite}

Assuming the glass beads are homogeneously dispersed in PEK matrix, the rule of mixtures were applied to simulate the CTE of glass beads filled PEK composite as shown in equation (4) and (5)

$$
\begin{aligned}
& \alpha_{L S-G l a s s / P E K}=V_{G B} \times \alpha_{G B}+\left(1-V_{G B}\right) \times \alpha_{L S-P E K} \\
& V_{G B}=W_{G B} /\left[W_{G B}+\left(1-W_{G B}\right) \times \rho_{G B} / \rho_{L S-P E K}\right]
\end{aligned}
$$

Where $V_{G B}$ and $W_{G B}$ represents the volume and weight percentage (20\%) of glass beads. $\rho_{G B}$ and $\rho_{L S-P E K}$ are the densities of glass beads $\left(2.5 \mathrm{~g} \mathrm{~cm}^{-3}{ }^{[30]}\right)$ and LS-PEK $(1.3$ $\left.\mathrm{g} \mathrm{cm}^{-3}{ }^{[33]}\right)$, respectively. The $V_{G B}$ is calculated as $0.12 . \alpha_{G B}$ and $\alpha_{L S-P E K}$ are the CTEs of glass beads $\left(9 \mathrm{ppm}{ }^{\circ} \mathrm{C}^{-1}{ }^{[30]}\right)$ and LS-PEK, respectively. The measured $\alpha_{L S-P E K}$ is used into the calculation of $\alpha_{L S-G B / P E K}$.

Figure 7 presents the calculated and measured CTE of LS-GB/PEK composite below and above Tg in $\mathrm{X}, \mathrm{Y}$, and $\mathrm{Z}$ directions. In Figure 7, the simulated CTE values were considered for composites incorporating up to $30 \%$ glass (by volume), as it is suspected that higher percentage of glass will lead to weaker composite structures, especially when using laser sintering as manufacturing technique. The measured value matches the simulated CTE below Tg. Simulation curves show the CTE in Z direction is lower than that in $\mathrm{X}$, Y direction. The CTEs between $\mathrm{Z}$ and $\mathrm{X}$, Y directions show more difference at temperature above $\mathrm{Tg}$ than below $\mathrm{Tg}$. Above $\mathrm{Tg}$, the simulated CTE is 
approximately 5\% higher than the measured CTE value in each direction. This is not surprising as above $\mathrm{Tg}$ there is a higher degree of molecular movement.

\section{Conclusions}

This study achieved, for the first time, manufacture of GB/PEK composites using high temperature laser sintering system. In comparison with the laser sintered PEK parts, the LS-GB/PEK composite had an improved hardness (7\% increase) and reduced CTEs (9$12 \%$ reduction below $\mathrm{Tg}$ and $15-16 \%$ reduction above the $\mathrm{Tg}$ ), without reducing its tensile strength.

The tensile strength of LS-PEK was slightly lower than that of IM-PEK parts. This could be related with multi-layered microstructure of LS-PEK. The presence of particle layers in LS-PEK suggests that the full molecular coalescence was not achieved whereas in injection moulding, the polymers were homogeneously and fully melted at molecular level. Hence, IM-PEK had better mechanical strength than LS-PEK. Addition of $20 \mathrm{wt} . \%$ glass beads had no negative effect on the tensile strength.

The laser sintering process led to parts with higher crystallinity than the injection moulded parts. The difference in Vickers hardness of PEK structures manufactured by the two methods originated from the variation in their crystallinity and lamellar thickness of crystals which was due to differences in thermal history arising from differences in the thermal cooling cycles.

The thermal expansion of LS-PEK and LS-GB/PEK composite was anisotropic. The CTE values in $\mathrm{Z}$ direction are lower than those in the $\mathrm{X}$ and $\mathrm{Y}$ directions. This is related to the multi-layered structure. Rule of mixture is applied to simulate the thermal expansion of LS-GB/PEK composite. Below the Tg, the experimental measurements of 
the composite match the rule of mixture calculations, whereas above the $\mathrm{Tg}$, the simulated and experimental values are diverging slightly.

\section{Reference}

[1] Jones DP, Leach DC and Moore DR. Mechanical Properties of Poly(ether-etherketone) for engineering. Polymer 1985; 26: 1385-93.

[2] Blundell DJ and Osborn BN. The Morphology of Poly(aryl-ether-ether-ketone). Polymer 1984; 25: 953-8.

[3] Wang ZQ, Gao DR. Friction and wear properties of stainless steel sliding against Polyetheretherketone and carbon-fiber-reinforced Polyetheretherketone under natural seawater lubrication. Mater. Design 2014; 53: 881-7.

[4] Lee Y, Porter RS. Effects of thermal history on crystallization of Poly(ether ether ketone) (PEEK). Macromolecules 1988; 21: 2770-6.

[5] Zhai T, Di L, Yang D. Study on the pretreatment of Poly(ether ether ketone)/ multiwalled carbon nanotubes composites through environmentally friendly chemical etching and electrical properties of the chemically metallized composites. ACS Appl. Mater. Interfaces 2013; 5: 12499-509.

[6] Ho RM, Cheng SZD, Hsiao BS, Gardner KH. Crystal morphology and phase identifications in Poly(ary1 ether ketone)s and their copolymers. 1. polymorphism in PEKK. Macromolecules 1994; 27: 5787-93.

[7] Lee Y, Porter S. Crystallization of Poly(ether ether ketone) oriented by solid-state extrusion. Macromolecules 1991; 24: 3537-42. 
[8] J. Altmeyer J, dos Santos JF, Amancio-Filho ST. Effect of the friction riveting process parameters on the joint formation and performance of Ti alloy/short-fibre reinforced polyether ether ketone joints, Mater. Design 2014; 60:164-6.

[9] Kurtz SM, Devine JN. PEEK biomaterials in trauma, orthopedic, and spinal implants, Biomaterials 2007; 28: 4845-69.

[10] Toth JM, Wang M, Estes BT, Scifert JL, Seim HB, Turner AS.

Polyetheretherketone as a biomaterial for spinal applications. Biomaterials 2006; 27: 324-34.

[11] Hamada H, Ramakrishna S. Scaling effects in the energy absorption of carbonfibre/PEEK composites tubes. Compos. Sci. Technol. 1995; 55: 211-21.

[12] Engineering materials magazine, Thermoplastics to revolutionise the composite industry. $<\mathrm{http}: / / \mathrm{www}$.materialsforengineering.co.uk/engineering-materialsfeatures/thermoplastics-to-revolutionaise-the-composites-industry/52151>; June 2013. [13] High performance compsoties magzine, Thermoplastic composites : primary structure ? <http://www.compositesworld.com/articles/thermoplastic-compositesprimary-structure>; May 2011.

[14] Bassett K, R. Carriveau R, Ting SKD. 3D printed wind turbines part 1: Design considerations and rapid manufacture potential. Sustainable Energy Technologies and Assessments 2015; In Press.

[15] Strom P. Dynamic vehicle weight reduction and safety enhancement. SAE Int J Passeng. Cars-Mech. Syst.2009; 1: 1202-7. 
[16] Cooper D, Thornby J, Blundell N, R. Henrys R, Williams MA, Gibbons G, Design and manufacture of high performance hollow engine valves by additive layer manufacturing, Mater. Design 2015; 69: 44-5.

[17] Barnes JA, Simms IJ, Farrow GJ, Jackson D, Wostenholm G, Yates B. Thermal expansion characteristics of PEEK composites. J. Mater. Sci. 1991, 26, 2259-71.

[18] Lu ZP, K. Friedrich K. On sliding friction and wear of PEEK and its composites. Wear 1995; 181-183: 624-31.

[19] Choy CL, Leung WP, Nakafuku C. Thermal Expansion of Poly (ether-ether-ketone) (PEEK). J. Polym. Sci., Part B: Polym. Phys. 1990; 28: 1965-77.

[20] Voss H, Friedrich K. On the wear behaviour of short fibre reinforced PEEK composites. Wear 1987; 116: 1-18.

[21] Sinmazcelik T, Y1lmaz T, Thermal aging effects on mechanical and tribological performance of PEEK and short fiber reinforced PEEK composites. Mater. Design 2007; 28:641.

[22] Wippl J, Schmidt HW, Giesa R. High temperature thermosets with a low coefficient of thermal expansion. Macromol. Mater. Eng. 2005; 290: 657-68.

[23] Ghita OR, James E, Evan KE. Physico-chemical behaviour of Poly (Ether Ketone) (PEK) in high temperature laser sintering (HT-LS). J. Mater. Process. Technol. 2014; 214: 969-78.

[24] Berretta S, Ghita OR, Evans KE. Morphology of polymeric powders in laser sintering (LS): from Polyamide to new PEEK powders. Eur. Polym. J. 2014; 59: 21829. 
[25] Kausch HH, Beguelin Ph, and Fischer M. Failure of particulate reinforced polymers. Mech. Compos. Mater. 2000; 36: 177-84.

[26] Stuart BH, Briscoe BJ. Scratch hardness studies of Poly(ether ether ketone). Polymer 1996; 37: 3819-24.

[27] Zarringhalam H, Majewski C, Hopkinson N. Degree of particle melt in Nylon-12 selective laser-sintered parts. Rapid Prototyping J. 2009; 15: 126-32.

[28] Ajoku U, Saleh N, Hopkinson N, Hague R, Erasenthiran P. Investigating mechanical anisotropy and end-of-vector effect in laser-sintered nylon parts. Proc. IMechE, Part B: J. Engineering Manufacture 2006; 220: 1077-86.

[29] Ghita OR, James E, Davies R, Berretta S, Singh B, Flint S, Evans KE, High temperature laser sintering: An investigation into mechanical properties and shrinkage characteristics of Poly (Ether Ketone) structures. Mater. Design 2014; 61: 124-32. [30] High Performance Solid Glass Polymer Additives, Engineered glass materials division, Technical Datasheet. Potters Industries, LLC.

[31] Dawson PC and Blundell DJ. X-ray data for poly(aryl ether ketones). Polymer 1980; 21: 577-8.

[32] Sarasua RJ, Remiro PM. Effects of thermal history on mechanical behavior of PEEK and its short-fiber composites, J. Polym. Compos. 1996; 17: 468-77. [33] Materials Datasheet of EOS high temperature polymer PEEK HP3, EOS, 2013. 
Figure 1. The building positions of the parts in the chamber

Figure 2. (a) SEM image of glass beads, (b) SEM image of the PEK powder and (c) Fracture surface of LS-GB/PEK composite

Figure 3. (a)The stress-strain curves and ultimate tensile strength (UTS) of IM-PEK, LS-PEK and LS-GB/PEK composite, (b) the Vickers hardness (HV) of IM-PEK, LSPEK and LS-GB/PEK composite

Figure 4. (a) Fractured surface of LS-PEK samples showing a multi-layered structure, (b), (c) and (d) high magnification images of the multi-layered structure, (e) schematic illustration of the multi-layered structured developed during the LS method.

Figure 5. The multi-layered structure of LS-PA12 revealed unmelted particle cores ${ }^{[25]}$ Figure 6. WAXD spectra of LS-PEK, LS-GB/PEK and IM/PEK

Figure 7. Measured and simulated CTE of LS-GB/PEK on X, Y and Z direction (a) below $\operatorname{Tg}(\alpha g)$ and $(b)$ above $\operatorname{Tg}(\alpha \gamma)$ 
Table 1. (a) Tensile strength, Vickers hardness, crystallinity\%, averaged lamellar thickness and CTE of PEK powder, IM-PEK, LS-PEK and LS-GB/PEK composite,(b) Peak position and FWHM of crystal planes of IM-PEK, LS-PEK and LS-GB/PEK

\begin{tabular}{|c|c|c|c|c|c|c|}
\hline \multicolumn{7}{|l|}{ (a) } \\
\hline & \multicolumn{2}{|c|}{ IM-PEK } & \multicolumn{2}{|c|}{ LS-PEK } & \multicolumn{2}{|c|}{ LS-GB/PEK } \\
\hline Tensile Strength (MPa) & \multicolumn{2}{|c|}{$97.7 \pm 0.85$} & \multicolumn{2}{|c|}{$87.6 \pm 7.2$} & \multicolumn{2}{|c|}{$89.5 \pm 5.1$} \\
\hline Vickers Hardness & \multicolumn{2}{|c|}{$24.9 \pm 0.5$} & \multicolumn{2}{|c|}{$30.2 \pm 0.4$} & \multicolumn{2}{|c|}{$32.6 \pm 0.5$} \\
\hline Crystallinity\% (XRD) & \multicolumn{2}{|l|}{40.2} & \multicolumn{2}{|l|}{53.3} & \multicolumn{2}{|l|}{51.6} \\
\hline Average lamellar thickness & \multicolumn{2}{|l|}{9} & \multicolumn{2}{|l|}{15} & \multicolumn{2}{|l|}{15} \\
\hline$\alpha_{\mathrm{g}-\mathrm{X}}\left(\mathrm{ppm}{ }^{\circ} \mathrm{C}^{-1}\right)$ & \multicolumn{2}{|c|}{$52.3 \pm 1.7$} & \multicolumn{2}{|c|}{$51.0 \pm 0.82$} & \multicolumn{2}{|c|}{$45.3 \pm 0.47$} \\
\hline$\alpha_{\mathrm{g}-\mathrm{y}}\left(\mathrm{ppm}{ }^{\circ} \mathrm{C}^{-1}\right)$ & \multicolumn{2}{|c|}{$49.3 \pm 0.94$} & \multicolumn{2}{|c|}{$51.0 \pm 0.82$} & \multicolumn{2}{|c|}{$45.0 \pm 0.82$} \\
\hline$\alpha_{\mathrm{g}-\mathrm{Z}}\left(\mathrm{ppm}^{\circ} \mathrm{C}^{-1}\right)$ & \multicolumn{2}{|c|}{$49.3 \pm 1.25$} & \multicolumn{2}{|c|}{$49.0 \pm 0.82$} & \multicolumn{2}{|c|}{$44.7 \pm 0.94$} \\
\hline$\alpha_{\mathrm{r}-\mathrm{X}}\left(\mathrm{ppm}{ }^{\circ} \mathrm{C}^{-1}\right)$ & \multicolumn{2}{|c|}{$138.3 \pm 0.94$} & \multicolumn{2}{|c|}{$138.7 \pm 2.36$} & \multicolumn{2}{|c|}{$116.3 \pm 0.47$} \\
\hline$\alpha_{\mathrm{r}-\mathrm{y}}\left(\mathrm{ppm}{ }^{\circ} \mathrm{C}^{-1}\right)$ & \multicolumn{2}{|c|}{$135.7 \pm 6.13$} & \multicolumn{2}{|c|}{$136.3 \pm 1.25$} & \multicolumn{2}{|c|}{$116.0 \pm 0.82$} \\
\hline$\alpha_{\mathrm{r}-\mathrm{z}}\left(\mathrm{ppm}{ }^{\circ} \mathrm{C}^{-1}\right)$ & \multicolumn{2}{|c|}{$141.0 \pm 0.00$} & \multicolumn{2}{|c|}{ 124. $0 \pm 2.45$} & \multicolumn{2}{|c|}{$105.3 \pm 3.30$} \\
\hline \multicolumn{7}{|l|}{ (b) } \\
\hline & \multicolumn{2}{|c|}{ IM-PEK } & LS-F & EK & LS- & B/PEK \\
\hline Crystal plane & $2 \theta$ & FWHM & $2 \theta$ & FWHM & $2 \theta$ & FWHM \\
\hline$[110]$ & 18.2 & 0.71 & 18.6 & 0.49 & 18.6 & 0.49 \\
\hline [111] & 20.1 & 1.07 & 20.5 & 0.60 & 20.5 & 0.65 \\
\hline [200] & 22.4 & 1.20 & 22.8 & 0.81 & 22.8 & 0.85 \\
\hline [211] & 28.1 & 1.46 & 28.6 & 0.84 & 28.6 & 0.74 \\
\hline
\end{tabular}




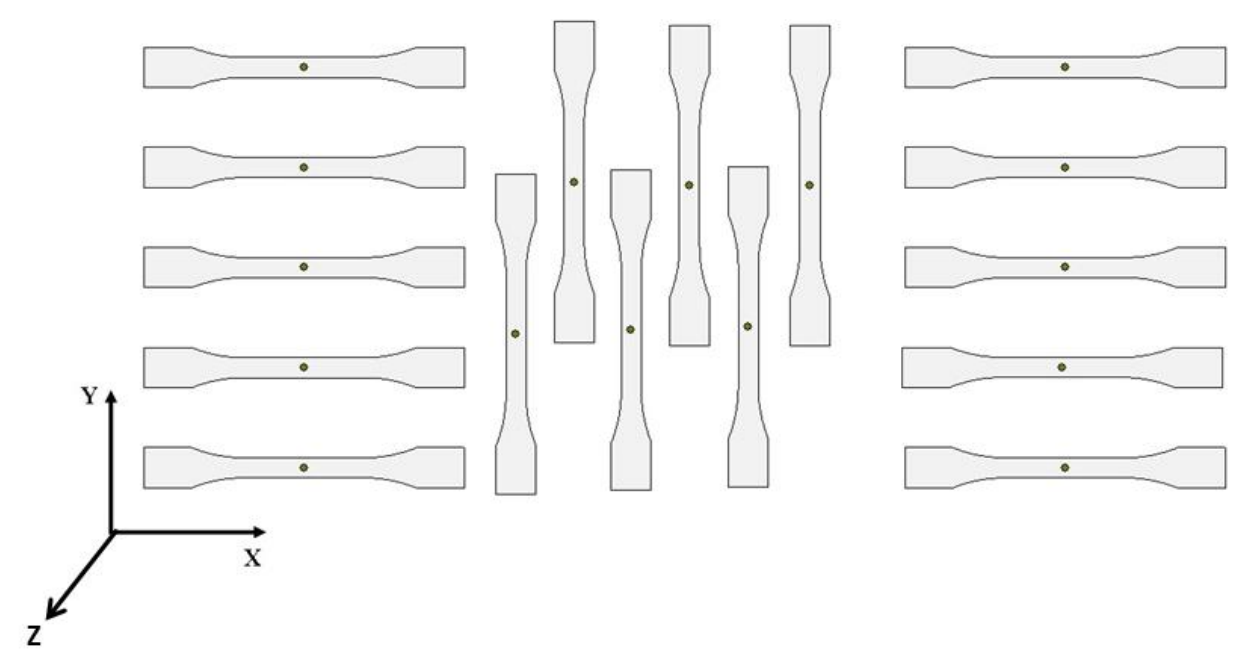

Figure 1. The building positions of the parts in the chamber
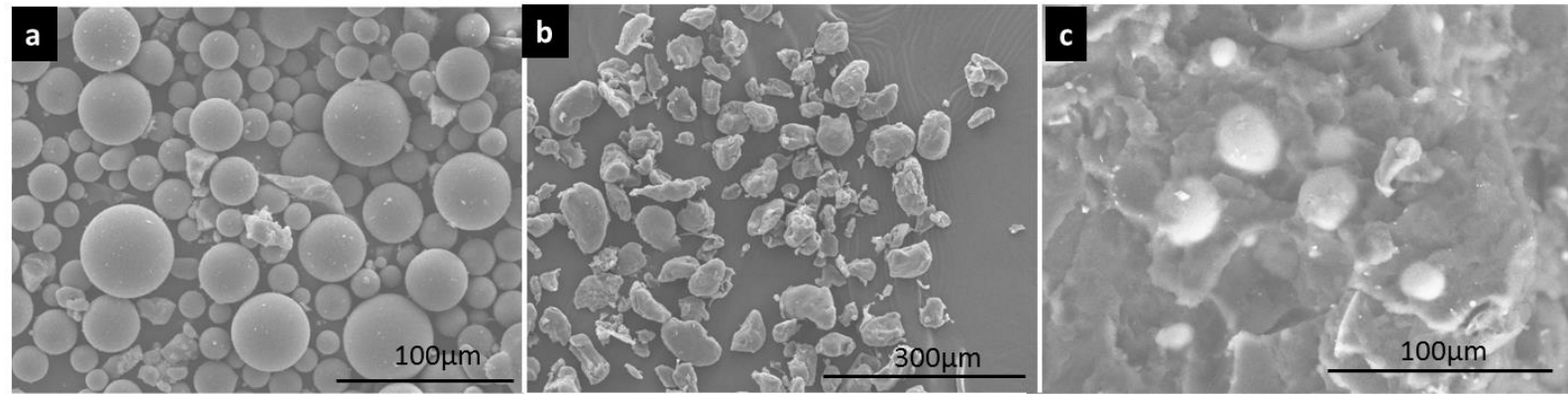

Figure 2. (a) SEM image of glass beads, (b) SEM image of the PEK powder and (c) Fracture surface of LS-GB/PEK composite 

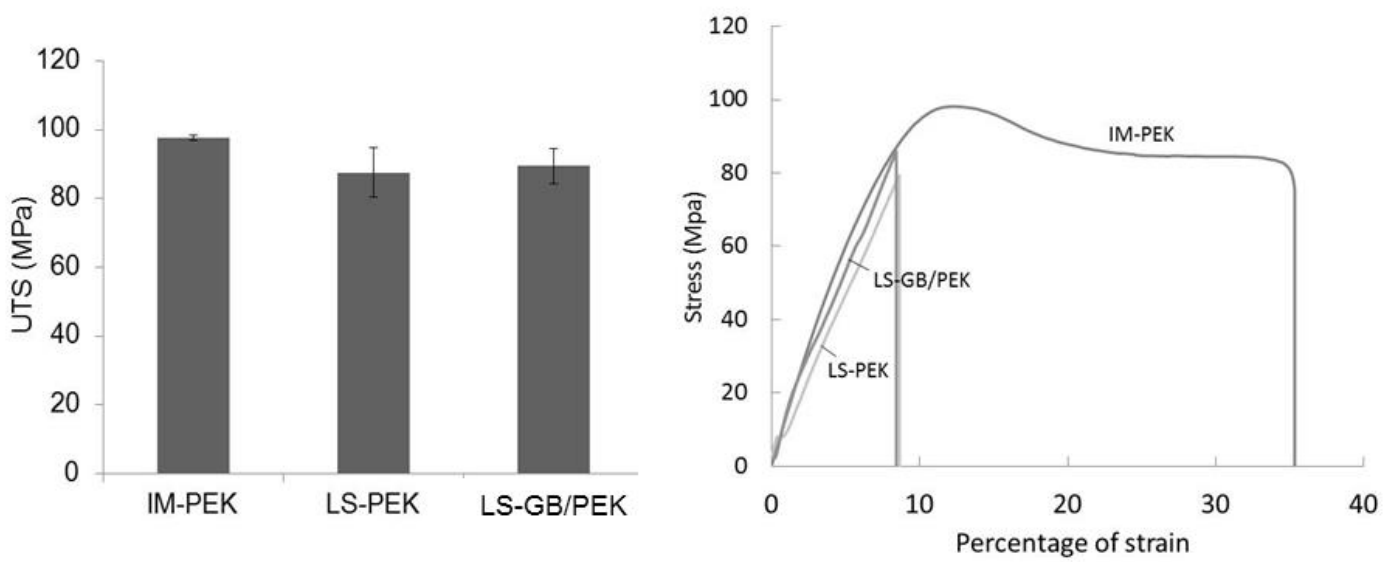

(a)

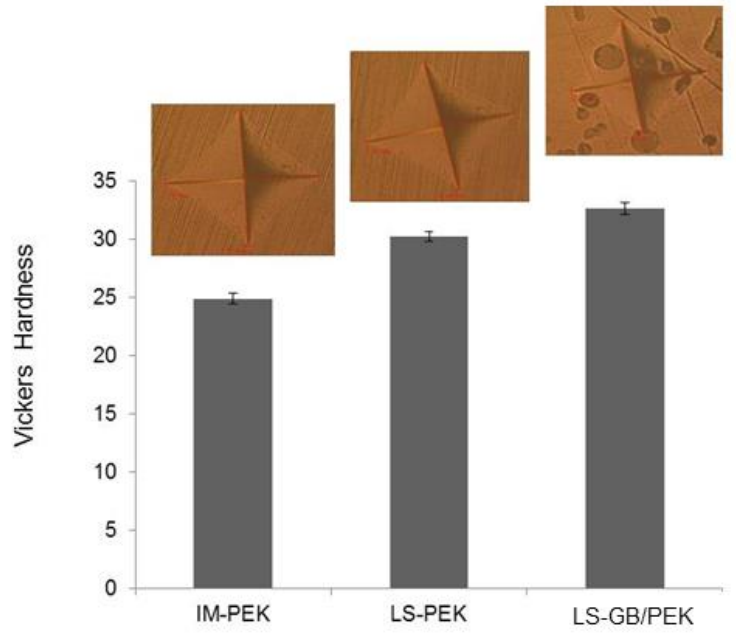

(b)

Figure 3. (a)The stress-stain curves and ultimate tensile strength (UTS) result of IM-PEK, LS-PEK and LS-GB/PEK composite, (b) the Vickers hardness (HV) of IM-PEK, LS-PEK and LS-GB/PEK composite 


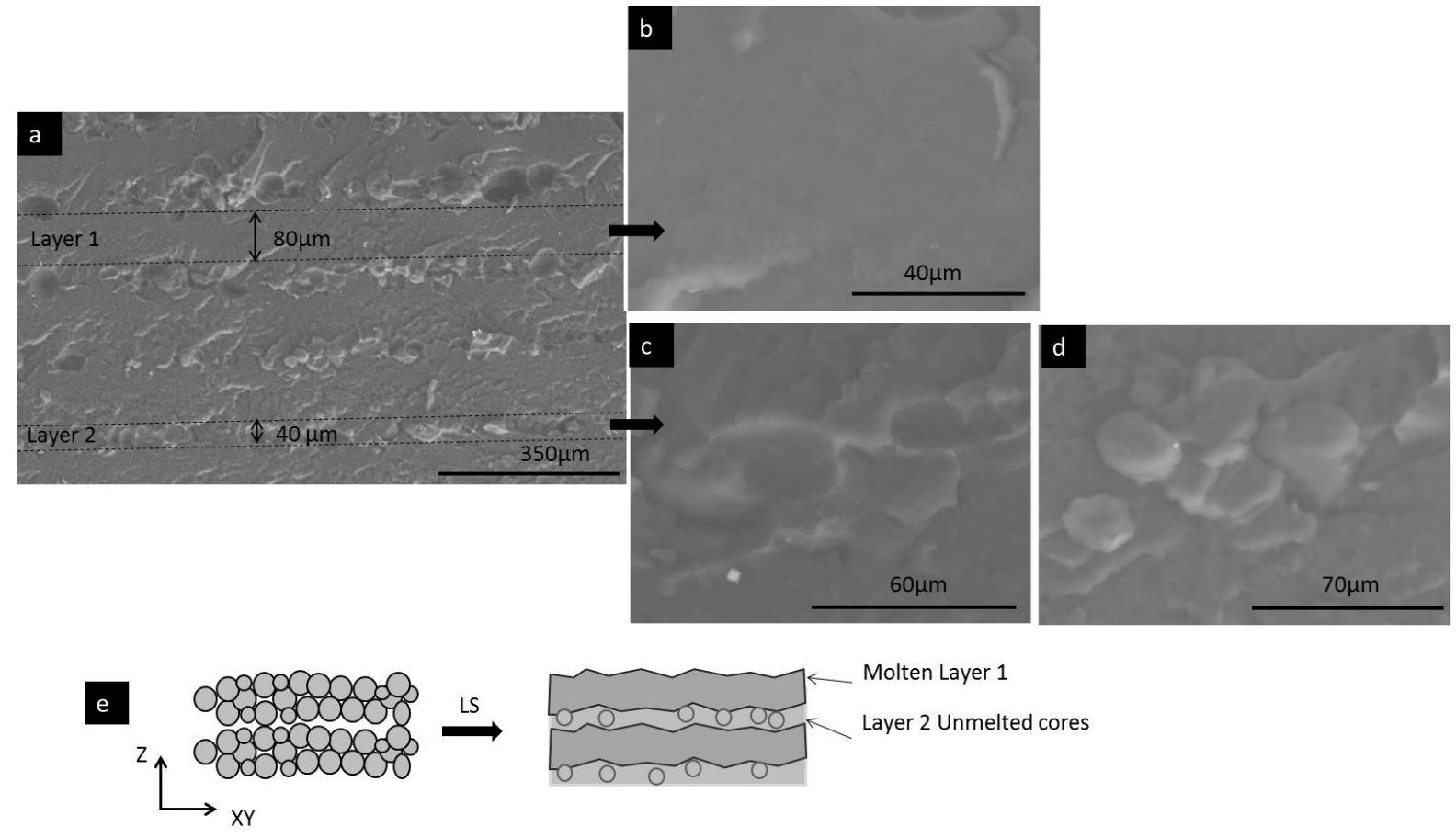

Figure 4. (a) Fractured surface of LS-PEK samples showing a multi-layered structure, (b),

(c) and (d) high magnification images of the multi-layered structure, (e) schematic

illustration of the multi-layered structured developed during the LS method. 


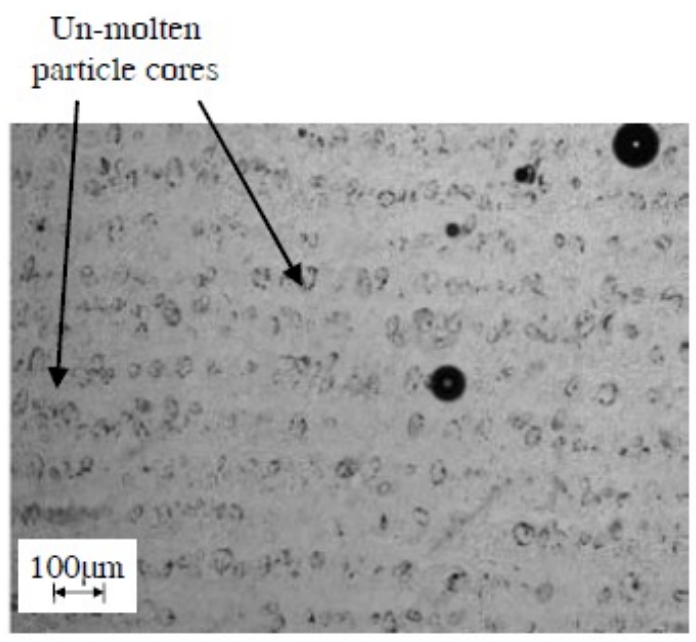

Figure 5. The multi-layered structure of LS-PA12 revealed unmelted particle cores $\left.{ }^{[27}\right]$

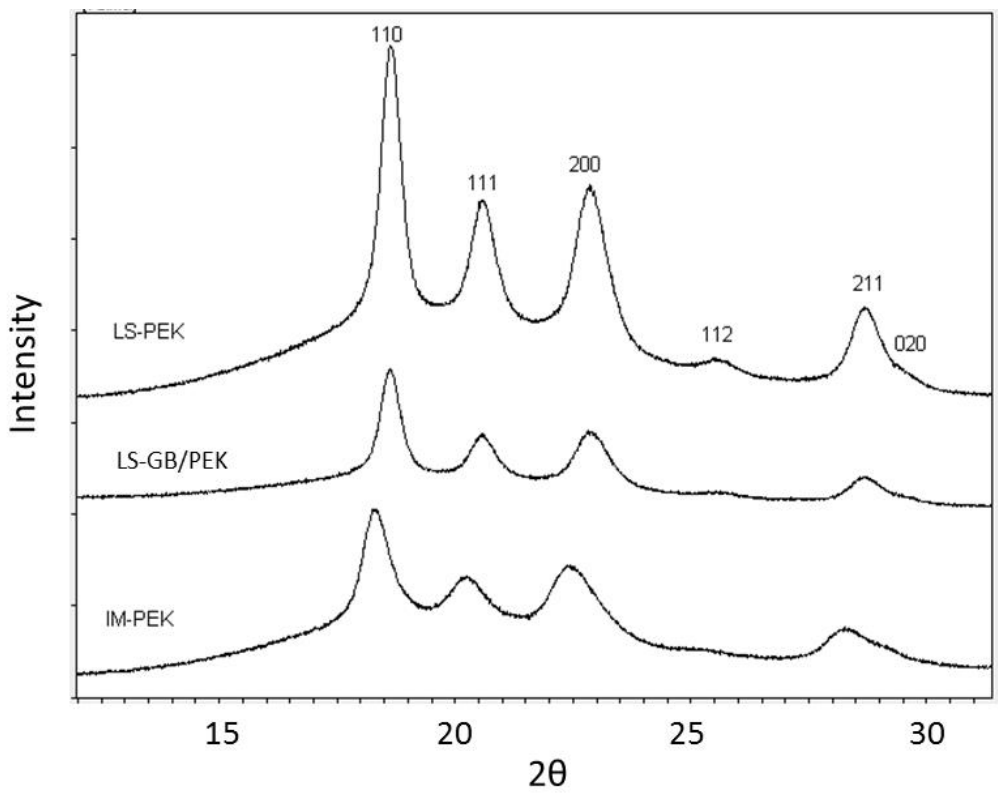

Figure 6. WAXD spectra of LS-PEK, LS-GB/PEK and IM/PEK 


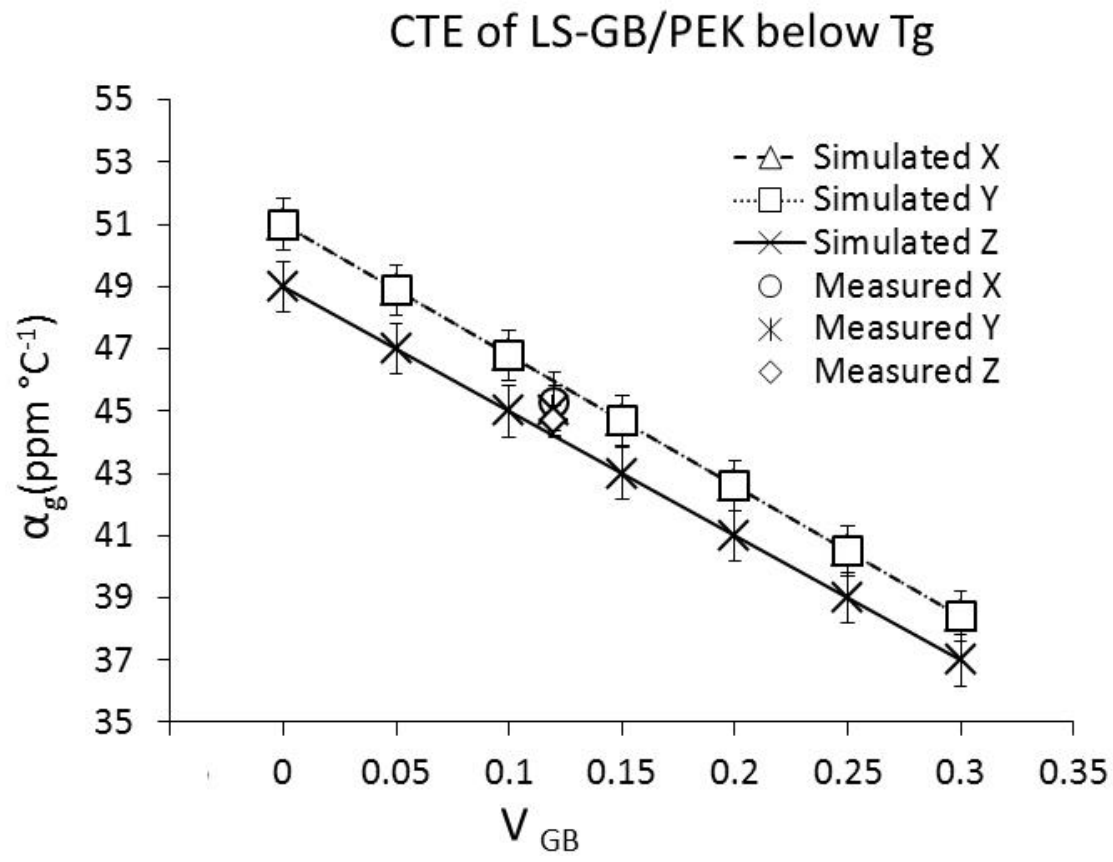

(a)

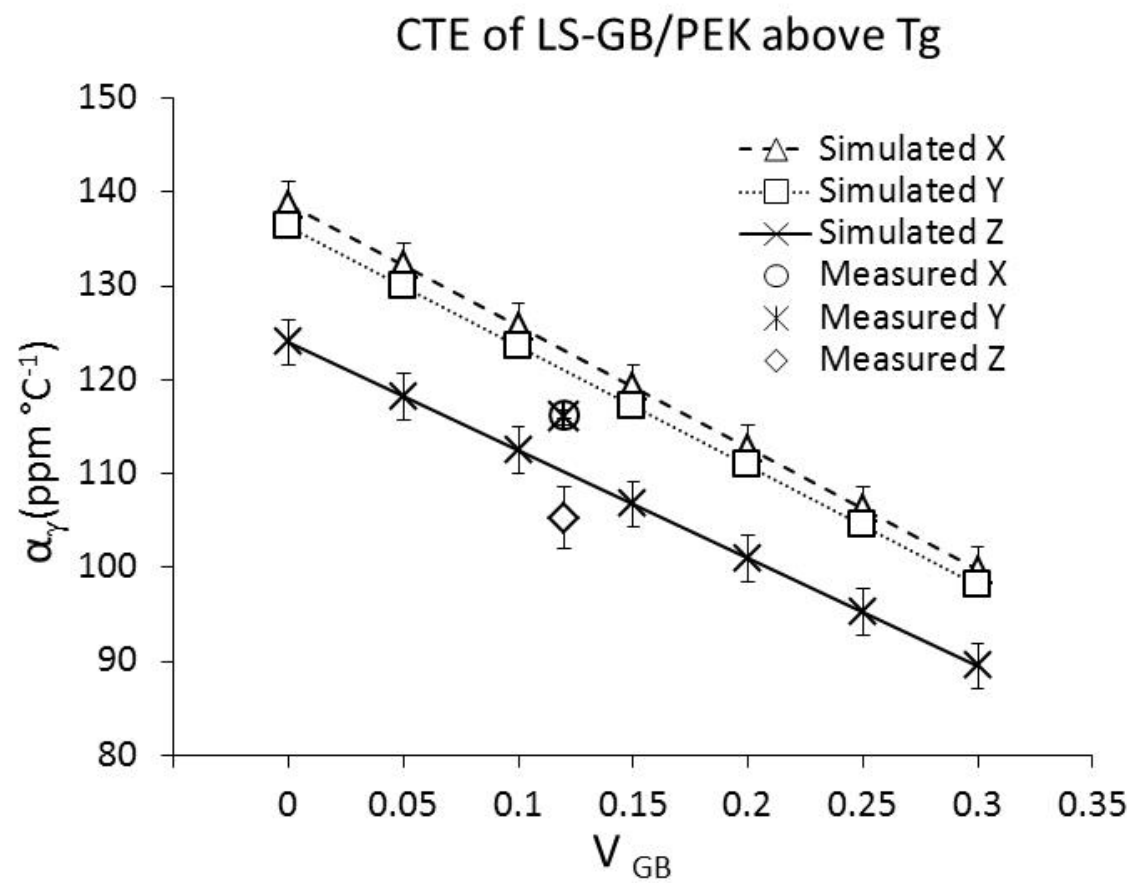

(b)

Figure 7. Measured and simulated CTE of LS-GB/PEK on X, Y and Z direction (a) below Tg $\left(\alpha_{g}\right)$ and $(b)$ above $\operatorname{Tg}\left(\alpha_{\gamma}\right)$ 